\title{
Molekulargenetische und physiologische Untersuchungen zur Vererbung des Erbdefektes Hernia inguinalis/scrotalis beim Schwein
}

\author{
Dissertation \\ zur Erlangung des Doktorgrades \\ der Fakultät für Agrarwissenschaften \\ der Georg-August-Universität Göttingen \\ vorgelegt von \\ Christian Beuermann \\ geboren in Göttingen
}

Göttingen, Juli 2009 
D 7

1. Referent: $\quad$ Prof. Dr. Dr. Bertram Brenig

2. Referent: Prof. Dr. Wolfgang Holtz

Tag der mündlichen Prüfung 16.07.2009 
Für die wichtigsten Menschen in meinem Leben: meine Frau Viola und meine Tochter Emma 
INHALT

Abstract I

Zusammenfassung II

Liste der Publikationen und Kongressbeiträge III

1 EINLEITUNG $\quad 8$

2 ENTSTEHUNG, KRANKHEITSBILD UND VERERBUNG VON HERNIA I/S 9

2.1 Kopplungs- und Assoziationsanalysen für Hernia i/s 10

3 DESCENCUS TESTIS $\quad 11$

3.1 Störung der Phasen des Descensus testis/Rolle des Gubernaculum testis 14

3.2 Obliteration des Processus vaginalis- mögliche Störungen 16

4 EINGLIEDERUNG DER ARBEIT IN DAS FORSCHUNGSPROJEKT 20

5 GENETIK UND PHYSIOLOGIE- QUO VADIS? 22

6 LITERATURVERZEICHNISS

$\begin{array}{llr}7 & \text { DANKSAGUNG } & 28\end{array}$

8 LEBENSLAUF 29

9 ANHANG $\quad 30$ 


\section{Abstract}

Hernia inguinalis/scrotalis (i/s) in pigs are known as congenital defects which occur with a frequency of $1-3 \%$ and a heritability of $h^{2}=0.02$ to $h^{2}=0.86$ in German pig breeds and cause enormous economic losses. Therefore, the elucidation of the mode of inheritance and the development of DNA based tests are of crucial economic and scientific concern.

At the Institute of Veterinary Medicine (IVM) the studies of the genetic basis of Hernia i/s take place within the national research project „Entwicklung von Techniken und Methoden zur Kartierung von Defektgenen beim Schwein".

In the present study, we determined the calcium contents in peritoneal tissue samples and samples of the Processus vaginalis of piglets which were associated with Hernia $i / s$. Our results showed a significant difference of the calcium content of tissue samples collected from the reproduction tract between the tissue samples obtained from affected animals compared to those of normal healthy pigs.

Due to these results - and additionally based on the results of a genome wide linkage scan, which was carried out at the IVM - we mapped functional candidate genes to porcine chromosomes (Sus scrofa chromosome, SSC) 3, 6, 7 and 15 which we additionally considered to be positional candidates. These candidate genes, which are supposed to be associated with Hernia i/s play an important role in the calcium homeostasis and within the apoptotic cascade. Furthermore, polymorphic microsatellites were detected within these candidate genes by target oligonucleotide mediated microsatellite identification (TOMMI) and tested in a population of affected pig families. 


\section{Zusammenfassung}

Bei Hernia inguinalis/scrotalis (i/s) des Schweins handelt es sich um einen kongenitalen Defekt, der mit einer Frequenz von 1-3\% und einer Heritabilität von $h^{2}=0,02$ bis $h^{2}=0,86$ in der Schweinezucht auftritt und zu hohen wirtschaftlichen Verlusten führt. Die Aufklärung der Vererbung dieses Defektes und die Entwicklung eines Gentestes sind somit von zentraler wirtschaftlicher und wissenschaftlicher Bedeutung. Am Tierärztlichen Institut der Universität Göttingen wird im Rahmen des Forschungsprojektes „Entwicklung von Techniken und Methoden zur Kartierung von Defektgenen beim Schwein" die molekulargenetische Untersuchung der Hernia i/s durchgeführt.

Im Rahmen dieser Arbeit wurde Tieren, die den Defekt Hernia i/s zeigten, Gewebeproben aus dem Processus vaginalis sowie dem peritonealen Gewebe entnommen und auf ihre Kalziumgehalte hin untersucht. Die Ergebnisse aus dieser Untersuchung haben gezeigt, dass die Kalziumgehalte in den untersuchten Geweben des Reproduktionstraktes sich von denen bei gesunden Tieren unterscheiden.

Darauf aufbauend wurden - basierend auch auf einer ebenfalls am Tierärztlichen Institut durchgeführten Kopplungs- und Assoziationsstudie - in der vorliegenden Arbeit auf den porcinen Chromosomen (Sus scrofa chromosome, SSC) 3, 6, 7 und 15 funktionell-positionelle Kandidatengene kartiert. Bei diesen Kandidatengenen, die mit dem Defekt Hernia i/s beim Schwein gekoppelt zu sein scheinen, wurden sowohl Gene aus der Kalziumhomöostase als auch apoptoseregulierende Gene berücksichtigt. Darüber hinaus wurden polymorphe Mikrosatelliten mittels "target oligonucleotide mediated microsatellite identification" (TOMMI) in diesen Kandidatengenen isoliert und an einer Population betroffener Familien typisiert. 


\section{Liste der Publikationen}

Die vorliegende Arbeit basiert auf den durch Fettdruck hervorgehobenen Publikationen:

1. Knorr, C., Beck, J., Beuermann, $C_{\text {, }}$ Chen, K., Ding, N. Gatphayak, K., Huang, L. S., Laenoi, W. \& Brenig, B. (2006): Chromosomal assignment of porcine oncogenic and apoptotic genes CACNA2D2, TUSC4, ATP2A1, COL1A1, TAC1, BAK1 and CASP9. Anim Genet, 37:523-525.

2. Knorr, C., Beuermann, C., Beck, J. \& Brenig, B. (2007): Characterization of the porcine multicopy ribosomal protein $\mathrm{SA} / 37-\mathrm{kDa}$ laminin receptor gene familiy. Gene, 395:135-143.

3. Beuermann, C., Germerodt, M., Beck, J., Brenig, B. \& Knorr, C. (2008): Chromosomal assignment of eight porcine genes involved in apoptosis. Anim Genet, 39:330-331.

4. Germerodt, M., Beuermann, C., Rohrer, G. A., Snelling, W. M., Brenig, B. \& Knorr, C. (2008): Characterization and linkage mapping of 15 porcine STS markers to fine-map chromosomal regions associated with hernia inguinalis/scrotalis. Anim Genet, 39:671-672.

5. Beuermann, C., Beck, J., Schmelz, U., Dunkelberg, H., Schütz, E., Brenig, B. \& Knorr, C. (2009): Tissue calcium content in piglets with inguinal or scrotal hernias or cryptorchidism. J Comp Path, 140:182-186.

\section{Kongressbeiträge:}

6. Knorr, C., Beuermann, C., Laenoi, W., Beck, J. \& Brenig, B.: Molecular decipherment of porcine Hernia inguinalis/scrotalis Proceedings of the $30^{\text {th }}$ ISAGConference, Porto Seguro, 20.-25.08.2006.

7. Beuermann, C., Beck, J., Chilla, A., Schmelz, U., Dunkelberg, H., Schütz, E., Brenig, B. \& Knorr, C.: Untersuchungen zum Kalziumgehalt verschiedener Gewebe bei Ferkeln mit Skrotal-/Inguinalhernien und Kryptorchismus (C18) GfT/DGfZ-Tagung Hohenheim, 26.-27.09.2007.

8. Germerodt, M., Beuermann, C., Beck, J., Schütz, E., Rohrer, G.A., Brenig, B. \& Knorr, C.: Approaching porcine Hernia inguinalis/scrotalis $2^{\text {nd }}$ European Conference on Pig Genomics (Pig Genome II), Ljubljana, Slovenia, June 4-5, 2008; editors P. Dovc et al.; ISBN: 978-961-6379-12-0; pp. 49-50.

9. Germerodt, M., Beuermann, C., Beck, J., Schütz, E., Rohrer, G.A., Brenig, B. \& Knorr, C.: Strategien zur Untersuchung angeborener Hodensack- und Leistenbrüche beim Schwein (B26) GfT/DGfZ-Tagung Bonn, 17.-18.09.2008. 


\section{Einleitung}

Innerhalb des vom Bundesministerium für Bildung und Forschung (BMBF) und vom Förderverein Biotechnologieforschung (FBF) geförderten nationalen Projektes „Entwicklung von Techniken und Methoden zur Kartierung von Defektgenen beim Schwein", in dem verschieden Anomalienkomplexe wie Afterlosigkeit, Spreizer, Gesäugeanomalien und Leisten- und Hodensackbrüche bearbeitet werden, erfolgten am Tierärztlichen Institut der Universität Göttingen molekulargenetische Untersuchungen zum Merkmalskomplex Leistenund Hodensackbrüche (Hernia inguinalis/scrotalis (i/s)).

Das Auftreten kongenitaler Defekte - vor allem von Hernia i/s - beim Schwein verursacht jährlich hohe wirtschaftliche Einbußen durch anfallende Behandlungen und geringere Tageszunahmen der Tiere, die je nach Frequenz in der deutschen Schweineproduktion - Schäden von ca. 9 Mio. $€$ verursachen (Knorr, 2005).

Methoden und Techniken zur molekularen Genomanalyse wurden entwickelt, um den Selektionserfolg in einer Population zu steigern. Für diese Selektionsverfahren kommt einerseits die Identifizierung der beteiligten (kausalen) Genvariante zum Tragen, andererseits kann bei komplex vererbten Erkrankungen und der Lokalisierung von QTL (quantitative trait loci) die Analyse dieser mittels gekoppelter Marker erfolgen.

Ziel der vorliegenden Arbeit war es, Genomregionen auf Chromosom 3, 6, 7 und 15 beim Schwein zu untersuchen und ggf. weiter einzugrenzen, die mit dem Merkmal Hernia i/s assoziiert sind (Bornemann-Kolatzki, 2004). Darüber hinaus erfolgten Untersuchungen zum Kalziumhaushalt spezieller Gewebe des Reproduktionstraktes bei Ferkeln mit Inguinalund Skrotalhernien, um die Ätiologie dieses Defektes weiter aufzuklären. 


\section{Entstehung, Krankheitsbild und Vererbung von Hernia i/s}

Bei Inguinal- und Skrotalhernien (Hernia inguinalis/scrotalis (i/s)) handelt es sich um angeborene kongenitale Defekte mit eindeutig heriditärer Genese, bei denen die betroffenen Tiere bereits seit der Geburt prädisponiert sind. Hernia i/s entstehen durch einen abnorm weiten Leistenkanal und/oder einen nicht obliterierten Processus vaginalis (PV) (Clarnette und Hutson, 1999, Waldmann und Wendt, 2001). Ein Leistenbruch (Hernia inguinalis) liegt vor, wenn Eingeweide, meist Teile des Dünndarms und des großen Netzes, in den Leistenkanal vorfallen. Ein Hodensackbruch (Hernia scrotalis) kann aus einer Inguinalhernie entstehen, wenn diese Eingeweide bis in das Skrotum vorfallen. Hernia i/s treten entweder einseitig oder beidseitig auf, wobei bei männlichen Schweinen meist linksseitige Hernienbildung beobachtet wurde (Stigler, 1990). Obwohl Ingunial- und Skrotalhernien in beiderlei Geschlecht beim Schwein detektiert wurden, konnte Heres in einer Studie ein sechsmal häufigeres Auftreten von Brüchen beim männlichen als beim weiblichen Tier feststellen (Heres, 1976).

Berge gelang es Mitte der zwanziger Jahre des letzten Jahrhunderts bereits in Rückkreuzungsversuchen die Erblichkeit von Hernia i/s zu zeigen (Berge, 1941). Der Erbgang ist allerdings bis dato nicht eindeutig geklärt und wird in der Literatur teils kontrovers diskutiert. Hernien werden wahrscheinlich nicht nur oligo- sondern polygen vererbt, das heißt, dass mehrere Mutationen in verschiedenen Genen für das Auftreten von Ingunial- und Skrotalhernien verantwortlich zu sein scheinen. Außerdem handelt es sich um oligofaktoriell bedingte Anomalien, bei denen neben genetischen auch Umwelteffekte eine Rolle spielen (Beißner, 2003).

Hernien treten mit einer Frequenz von $0,5 \%$ bis $1 \%$ in der deutschen Schweinezucht auf (Althoff et al., 1988). Heritabilitätsschätzungen wurden anhand verschiedener Modelle für unterschiedliche Schweinerasen kalkuliert. Dabei ermittelte Althoff die niedrigsten Schätzwerte $\left(h^{2}=0,022 \pm 0,006\right.$ bis $\left.0,035 \pm 0,007\right)$ in einem 
linearen Modell (Althoff, 1985). Die höchsten Heritabilitätsschätzungen $\left(\mathrm{h}^{2}=0,65 \pm 0,06\right.$ und $\left.0,86 \pm 0,16\right)$ wurden von Mikami und Fredeen in zwei verschiedenen Populationen ermittelt (Mikami und Fredeen, 1979). Buschbell ermittelte in einer jüngeren Studie Heritabilitäten von $h^{2}=$ 0,14 anhand eines linearen Modells (Buschbell et al., 2007).

Jüngst veröffentlichte Zahlen besagen, dass 2007 in Deutschland ca. 2,8 Mio. Sauen gehalten wurden, die durchschnittlich 23,5 Ferkel geboren haben. Geht man dabei von einer Häufigkeit von $0,5 \%-1 \%$ der Anomalie Hernia i/s aus, so sind 329.000 bis 658.000 Ferkel pro Jahr betroffen (ZDS, 2007).

Die meisten der sich mit der Vererbung von Hernia i/s befassenden Arbeiten beziehen sich auf die Verhältnisse beim männlichen Schwein (Bornemann-Kolatzki, 2004, Grindflek et al., 2006, Beck, 2008, Germerodt, 2009), wobei auch die vorliegende Arbeit keine Ausnahme bildet.

\subsection{Kopplungs- und Assoziationsanalysen für Hernia i/s}

Die Kopplungsanalyse basiert auf der genetischen Rekombination. Sie stellt ein Verfahren dar, bei der Genomregionen resp. Gene lokalisiert bzw. identifiziert werden, die mit einem bestimmten Merkmal/Defekt gekoppelt sind. Diese Methode findet vor allem bei monogenen Erbkrankheiten oder der Identifizierung von „Hauptgenen" einer komplexen Erbkrankheit ihren Einsatz. Die Kopplungsanalyse basiert auf den genetischen Rekombinationsereignissen, wobei zugrunde gelegt wird, dass genetische Marker in der Nähe eines Genortes oder Gens liegen und innerhalb von Familien gemeinsam mit diesen vererbt werden. Kopplungsanalysen bei landwirtschaftlichen Nutztieren werden, laut Rothschild und Kollegen, meist an großen Tierzahlen aus experimentellen $\mathrm{F}_{2^{-}}$Populationen getestet, wobei häufig sehr große QTL-Regionen nachgewiesen werden (Rothschild et al., 2007). Bei der Assoziationsanalyse, die eine höhere statistische Aussagekraft im Vergleich zur Kopplungsanalyse hat und daher auch kleinere Stichproben 
verlangt, handelt es sich um eine Methode, positive Ergebnisse aus der Kopplungsanalyse zu bestätigen. Sie wird genutzt, um statistische Zusammenhänge genetischer Polymorphismen und quantitativer und/oder qualitativer Merkmale aufzuklären. Bei genomweiten Kopplungs- und Assoziationsanalysen werden Marker genutzt und auf ihren Zusammenhang mit einem Merkmal untersucht. Bickeböller und Fischer gehen davon aus, dass eine gesicherte statistische Assoziation gegeben ist, wenn eine Genvariante ursächlich einem Krankheitsbild zugeordnet werden kann oder sich im linkage disequillibrium (LD) befindet (Bickeböller und Fischer, 2007).

Für die Defekte Hernia i/s wurden bis dato Daten aus zwei genomweiten QTL-Scans veröffentlicht, deren Ergebnisse jedoch nur minimale Überlappungen aufzeigten, was wiederum dafür spricht, dass es sich bei Hernia $i / s$, wie bereits erwähnt, um einen multifaktoriell bedingten Erbgang handelt. In beiden Studien wurde neben einer Kopplungs- auch eine Assoziationsanalyse durchgeführt. Bornemann-Kolatzki führte in ihrer Arbeit einen Genomscan mit polymorphen DNA-Markern und einem Genomic mismatch scanning durch, um die an einer Vererbung des Defektes Hernia i/s beteiligten Chromosomenregionen zu charakterisieren. Im Rahmen dieser Arbeit wurden fünf mit dem Defekt assoziierte Regionen auf den porcinen Chromosomen SSC3, SSC6, SSC7, SSC12 und SSC15 detektiert (Bornemann-Kolatzki, 2004). Im Verlauf des Forschungsprojektes sollen diese Chromosomenregionen weiter eingegrenzt und mögliche Mutationen in potentiellen Kandidatengenen untersucht werden. Dabei ist das Ziel dieser Arbeiten, einen Gentest zu entwickeln, mit dem es möglich sein wird, betroffene Tiere von der Zucht zu isolieren.

\section{Descensus Testis}

Unter dem Descensus testis (DT) versteht man die Verlagerung des Hodens aus seiner ursprünglichen Lage an der dorsalen Wand der Bauchhöhle bis in das Skrotum (Abb. 3.1). Der DT lässt sich in verschiedene Phasen untergliedern und wird in der Literatur als mehrphasiger Prozess beschrieben (Hutson, 1985). Der Autor 
unterscheidet beim DT zwischen der inneren (transabdominalen) und der äußeren (inguinoskrotalen) Phase der Wanderung. Amman und Veeramachaneni weisen weiterführend auf die Unterteilung der von Hutson beschriebenen äußeren Phase in eine transinguinale und eine inguinoskrotale Wanderung der Hoden hin (Amann und Veeramachaneni, 2007).

Die transabdominale Phase umfasst die Migration der Hoden durch das Abdomen bis vor den Inguinalring und den späteren Leistenkanal. Die transinguinale Phase beschreibt den Durchtritt der Hoden durch den inneren und äußeren Leistenring, die inguinoskrotale Phase hingegen die weitere Migration der Hoden bis ins Skrotum (Amann und Veeramachaneni, 2007).

Der DT erfolgt beim Schwein um den 90. Tag der Trächtigkeit und ist zum Zeitpunkt der Geburt bereits abgeschlossen.

Grundsätzlich ist der Verlauf des DT bei vielen Nutz- und Haustieren sowie dem Menschen ähnlich, jedoch bilden einige Spezies (z. B. Elefanten), bei denen die Hoden nicht absteigen bzw. Kaninchen, bei denen der PV nicht obliteriert, die Ausnahme (Amann und Veeramachaneni, 2007, Döpfer, 2009). Einen festen Verschluss des Leistenkanals und die Obliteration des PV findet man beim Schwein, Rind und beim Menschen (Williams und Hutson, 1991). 

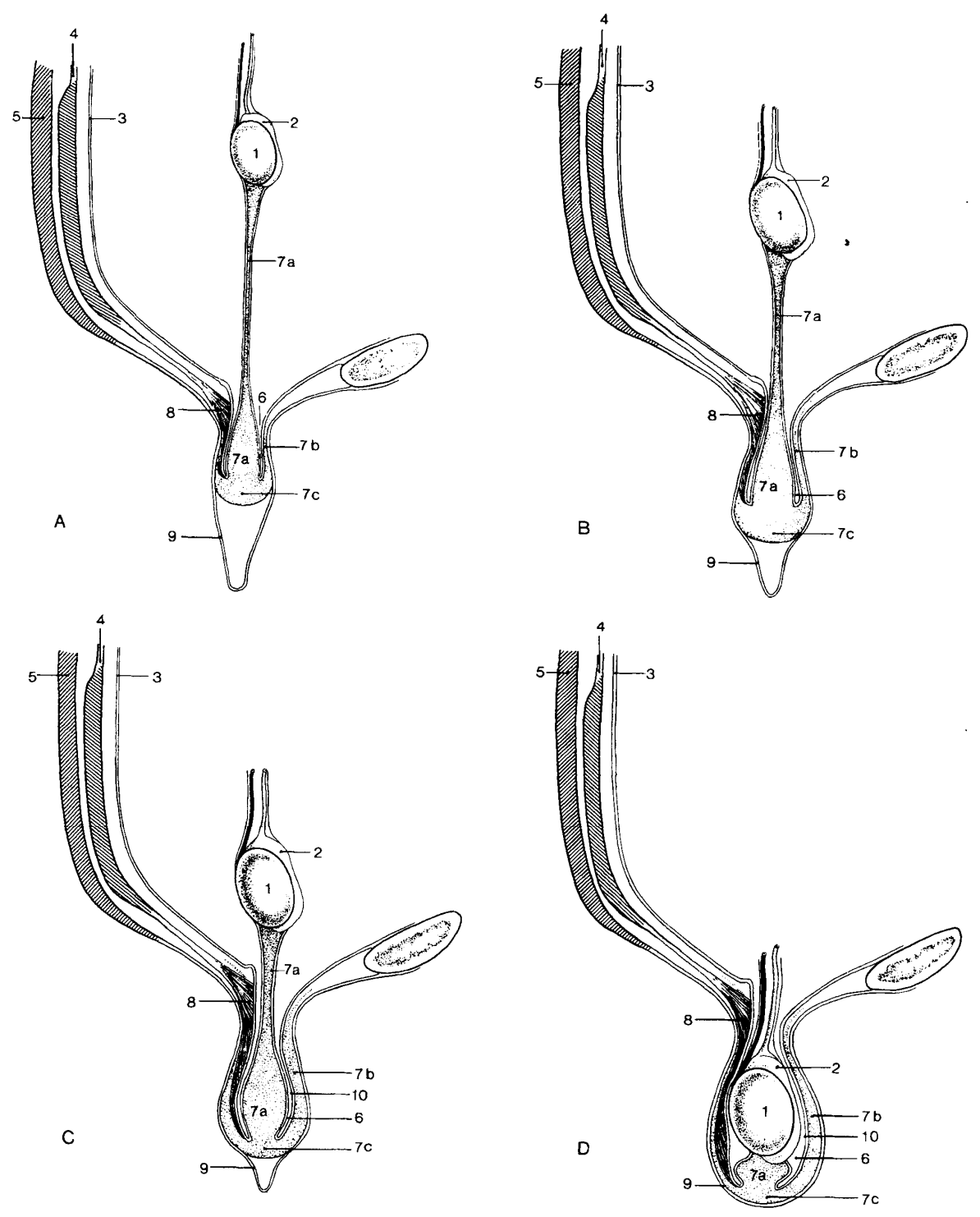

Abbildung 3.1: Descensus testis beim Schwein (Wensing, 1986).

(A) Entwicklung am Tag 65 post conceptionem (p.c.); (B) 75 Tage p.c.; (C) 85-90 Tage p.c.; (D) bei der Geburt. 1. Hoden (Testis); 2. Nebenhoden (Epididymis); 3. parietales Blatt des Bauchfells (parietales Peritoneum); 4. innerer schiefer Bauchmuskel (Musculus obliquus internus abdominis); 5. äußerer schiefer Bauchmuskel (Musculus obliquus externus abdominis); 6. Scheidenhautfortsatz (Processus vaginalis); 7a. Gubernaculum testis, 7b. vaginaler Teil (Pars vaginalis); 7c. Infravaginaler Teil (Pars infravaginalis); 8. Hodenheber (Musculus cremaster); 9. Fascia spermatica; 10. Tunica vaginalis. 


\subsection{Störung der Phasen des Descensus testis/Rolle des Gubernaculum testis}

Eine Störung des DT kann während allen drei der unter Punkt 3 beschriebenen Phasen auftreten, wobei in der Literatur diverse Hypothesen teils kontrovers diskutiert werden.

Adham beschreibt in seiner Arbeit den Einfluss des insulin-like growth factor oder insulin-like peptide hormone (INSL3) auf die Entwicklung des Gubernaculum testis in der transabdominalen Phase. Dabei wird INSL3 in den Leydigschen-Zwischenzellen gebildet (Adham et al., 2000). Bei Untersuchungen an "knock-out"-Mäusen konnte gezeigt werden, dass die Gubernacula testis klein und unterentwickelt waren und die Hoden parallel in ihrer abdominalen Lage verblieben (Nef und Parada, 1999, Zimmermann et al., 1999).

Der Einfluss von HOXA10 und HOXA11 auf die Entwicklung des Gubernaculum testis konnte noch nicht zweifelsfrei geklärt werden. Satokata konnte jedoch in $H O X A 10^{-}$Mäusen zeigen, dass dieses Gen auch zu einem intraabdominalen Verbleiben der Hoden führt (Satokata et al., 1995).

Beißner diskutiert in ihrer Arbeit die positive Korrelation zwischen den kongenitalen Defekten Kryptorchismus und Hernia i/s. Hierbei wurden große rassenspezifische Unterschiede der additiv-genetischen Korrelation der beiden Defekte gefunden. In Pietrain-Linien konnten geringe positive $\left(r_{g}=0,12\right)$ additiv-genetische Korrelationen nachgewiesen werden, wohingegen in Deutsche Landrassse-Linien negative Korrelationen $\left(r_{g}=-0,39\right)$ gefunden wurden (Beißner, 2003). Inwieweit ein gestörter DT ursächlich an dem Auftreten beider Anomalien beteiligt ist, lässt sich nicht eindeutig klären. Beck geht in ihrer Arbeit davon aus, dass keiner der Defekte monofaktoriellen Ursprung hat. Vielmehr verweist sie auf den heterogenetischen Einfluss verschiedener Mutationen in unterschiedlichen Genen sowie deren Wirkung auf unterschiedliche Phasen des DT. Gene, die in unterschiedlichen Phasen des DT wirken, müssen nicht kausal mit der Ausprägung beider Phänotypen in Zusammenhang stehen (Beck, 2008). 
Assoziationsstudien beim Schwein haben gezeigt, dass sowohl HOXA10 als auch INSL3 in der untersuchten Population keinen Einfluss auf die Ausbildung bzw. die Entstehung von Hernia i/s haben (Uibeleisen, 2001, Knorr et al., 2004).

Amann und Veeramachaneni gehen davon aus, dass Hernia i/s als Folge von transinguinalem Kryptorchismus auftreten können. Wahrscheinlicher erscheint jedoch eine gestörte äußere Phase der Migration des Testis, die zu einem offenen Inguinalkanal und/oder zu einem nicht vollständig obliterierten PV und damit zu Hernia i/s führt.

Das Gubernaculum testis nimmt während der transinguinalen bzw. inguinoskrotalen Phase des DT eine zentrale Position ein. Während der Migration des Hodens zeigen sowohl das Gubernaculum testis als auch der PV ein enormes Wachstum, welches beim Gubernaculum testis auf die Einlagerung von Wasser, durch die Bildung von Hyaluronsäure, zurückzuführen ist (Heyns et al., 1990, Cain et al., 1994). Hutson und Wensing beschreiben in ihren Arbeiten den zunehmenden intraabdominalen Druck, der neben der Größenreduktion des Hodens kurz vor dem Durchtreten durch den Leistenkanal, diesen schließlich durch den Inguinalkanal schiebt (Wensing, 1988, Hutson et al., 1997). Bereits während der transinguinalen Phase des DT bildet sich das Gubernaculum testis zurück, bedingt durch die katabole Wirkung verschiedener Enzyme, wie Hyaluronidase (HYAL) und $\beta$-Glucuronidase (GUSB) auf die Hyaluronsäure (Levy und Husmann, 1995). Beck und Gatphayak haben in ihren Arbeiten den möglichen Einfluss von GUSB und HYAL auf die Ausbildung von Hernia i/s untersucht und konnten jedoch einen kausalen Zusammenhang für Hernia i/s ausschließen (Gatphayak et al., 2004, Beck et al., 2006).

Eine zentrale Rolle spielt Testosteron aus den LeydigschenZwischenzellen für den Hodenabstieg während der inguinoskrotalen Phase. Eine direkte Auswirkung auf die vorherigen Phasen im DT konnten aber nicht nachgewiesen werden (Heyns et al., 1990). McMahon konnte an porcinen Feten zeigen, dass Testosteron nicht direkt auf die inguinoskrotale Migration des Hodens wirkt, sondern 
bereits während der transabdominalen Phase zur Maskulinisierung des gentifemoralen Nervs (GFN) führt (McMahon et al., 1995).

Hutson und Hasthorpe beschreiben eine Beteiligung des calcitonin generelated peptide (CGRP) während des DT. Durch die o. g. Maskulinisierung der Zellen wird CGRP ausgeschüttet bzw. die Sekretion erhöht. Dies führt zu einer rhythmischen Kontraktion des Gubernaculum testis in Richtung Skrotum und somit zum DT (Hutson und Hasthorpe, 2005).

\subsection{Obliteration des Processus vaginalis - mögliche Störungen}

Die Obliteration des PV spielt eine zentrale Rolle in Bezug auf das Auftreten von Hernia i/s (Clarnette et al., 1998), wobei der PV nur dann vollständig obliteriert, wenn der Hoden seine endgültige Lage im Skrotum - nach der inguinoskrotalen Migration - erreicht hat (Barthold und Redman, 1996).

Die wichtige Bedeutung des Testosterons für den Hodenabstieg wurde bereits unter Punkt 3.1 aufgezeigt (McMahon et al., 1995). Eine jüngere Studie verweist auf die Assoziationen verschiedener Polymorphismen des CAG-repeats im ersten Exon des Androgen Rezeptors $(A R)$. Hierbei wurde im Phänotyp ein einseitiger Kryptorchismus mit einem offenen PV detektiert, was die Bedeutung der Androgene und des AR für den DT und die Obliteration des PV bekräftigt (Silva-Ramos et al., 2006). Hutson und Kollegen untersuchten die Wirkung von CGRP auf die Fusion des PV-Epithels an menschlichen Organkulturen, wobei sie zeigen konnten, dass es zu einer Transformation der Epithelzellen zu Fibroblasten während der Obliteration des PV kommt (Hutson et al., 2000). Tez konnte diese Ergebnisse in einer jüngeren Studie in einem Stammzellmodell bestätigen (Tez und Kilic, 2006).

Tanyel und Kollegen gehen hingegen davon aus, dass der Hoden nicht zuletzt in seiner inguinoskrotalen Phase durch eine "Triebkraft" der Muskelzellen aktiv bis in das Skrotum befördert wird (Tanyel und Okur, 2004). Der PV bildet hierbei nicht nur eine bloße Ausstülpung des 
umgebenen Peritoneums sondern vielmehr ein strukturiertes Gewebe, welches glatte Muskelzellen (SM) enthält (Ulusu et al., 2007).

Einen völlig anderen Weg der Argumentation zur Obliteration des PV bzw. auch zum DT gehen Tanyel und Kollegen. Hierbei spielt nicht, wie bei den meisten zuvor zitierten Publikationen, die "passive" Migration des Hodens bis ins Skrotum eine zentrale Rolle, sondern die "aktive" Kontraktion der glatten Muskelzellen entlang des Gubernaculum testis eine Rolle. Nach erfolgtem DT obliteriert der PV durch den programmierten Zelltod (PCD) bzw. die Apoptose der Muskelzellen (Tanyel et al., 2002). Dieser PCD wird, laut Tanyel und Kollegen, durch die Senkung des sympathischen Tonus und die relative Erhöhung des parasympathischen Tonus induziert. Eine Störung innerhalb des Ablaufes des PCD kann somit zu einem nicht obliterierten resp. peristent offenen PV führen (Tanyel und Okur, 2004).

Für eine erfolgreiche Obliteration des PV, nach abgeschlossenem DT, ist es von entscheidender Bedeutung, dass diese SM-Zellen der Apoptose unterliegen. Der PCD wird durch eine Innervation induziert, bei der der, wie bereits angesprochen, symphatische Tonus gesenkt und der parasympathische Tonus angehoben wird (Tanyel und Okur, 2004). Dabei haben Untersuchungen beim Menschen gezeigt, dass bei Patienten mit Inguinalhernien bzw. Kryptorchismus diese nervale Steuerung gestört ist und bei letzteren eine anhaltende Erhöhung des parasympathischen Tonus und eine erhöhte Menge an zytosolischem Kalzium vorlag (Ulusu et al., 2007). In einer älteren Arbeit fanden Tanyel und Kollegen bereits heraus, dass der Kalziumgehalt im Bruchsackgewebe bei Probanden mit Inguinalhernien (beiderlei Geschlechts) signifikant geringer war als der Kalziumgehalt bei Kryptorchiden (Tanyel et al., 2002).

Der Kalziumgehalt scheint eine zentrale Stellung einzunehmen, wenn es darum geht, Unterschiede in Geweben von Hernia i/s zu analysieren. Darüber hinaus konnte die Rolle des Kalziums im PCD bzw. in der zellulären Signalkette der Apoptose nachgewiesen werden (Rizzuto et al., 2003). 
Unter physiologischen Bedingungen wird Kalzium in der Zelle im Endoplasmatischen- (ER) bzw. Sarkoplasmatischen Retikulum (SR, Abb. 4.1) gespeichert (Demaurex und Distelhorst, 2003) und ist an einer Vielzahl zellulärer Prozesse beteiligt (Berridge et al., 2003). Bergner und Huber gehen in ihrer Arbeit auf die Kalziumkonzentrationen innerhalb und außerhalb der Zellen beim Menschen ein. Hierbei beschreiben sie ein zytosolisches Kalziumruhepotential von 100 nM während sie intrazelluläre Kalziumgehalte in mikromolaren Bereich nachweisen konnten. Weiterhin weisen sie darauf hin, dass Kalzium durch seine heterogene Beteiligung an fast allen zellulären Prozessen einen weitreichenden Einfluss auf die Physiologie in der Zelle hat (Bergner und Huber, 2008). Während der Apoptose fungiert Kalzium als einer der Hauptauslöser der Apoptosekaskade, was Zhong und Kollegen in ihrer Arbeit zeigten (Zhong et al., 2006).

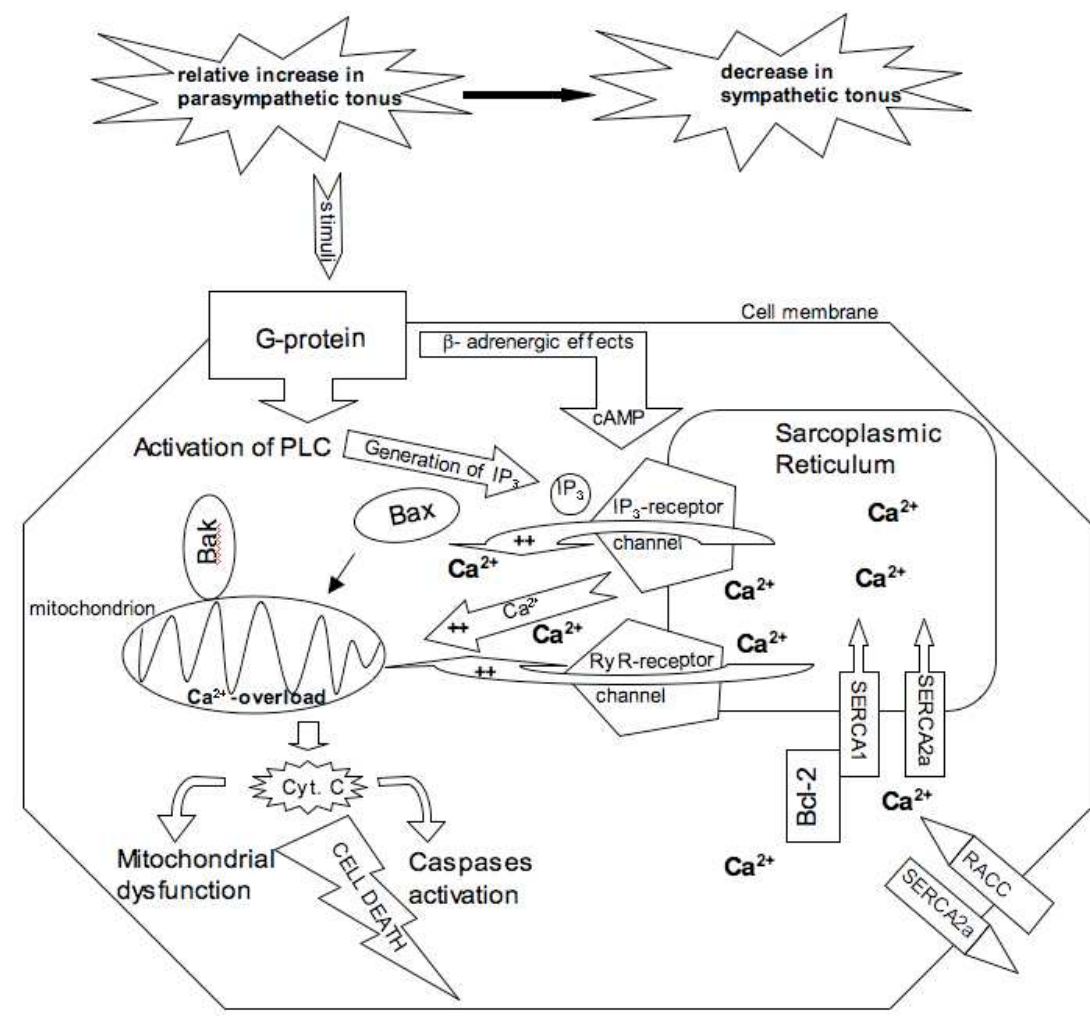

Abbildung 4.1: Schematische Darstellung der Apoptosekaskade. Die Apoptose wird über die Aktivierung von Phospholipase C (PLC) und die Generierung des inositol-1,4,5-posphat-receptor $\left(\mathrm{IP}_{3} \mathrm{R}\right)$ induziert. Über $\mathrm{IP}_{3} \mathrm{R}$ und den ryanodine receptor (RyR) wird Kalzium aus dem SR/ER ausgelagert. BCL2-associated $X$ protein (BAX) und BCL2-antagonist/killer (BAK) wirken proapoptotisch auf die Mitochondrien und somit auf die Sekretion von Cytochrom C. 
Lipskaia und Kollegen konnten jüngst zeigen, dass eine kurzzeitige Erhöhung des zytosolischen Kalziumgehaltes zu einer Erhöhung der physiologischen Kalziumkonzentration in den Mitochondrien führt, was wiederum zur Apoptose führt (Lipskaia et al., 2009). Diese resultiert aus einer Steigerung der Permeabilität und nicht zuletzt aus einer vermehrten Cytochrom C Freisetzung (Abb. 4.1, (Mattson und Chan, 2003, Deniaud et al., 2008)). Cytochrom C verstärkt die Kalziumsekretion aus dem ER/SR und führt zur Aktivierung von Caspase 9 und somit zur Apoptose (Orrenius et al., 2003).

Die Erhöhung der Kalziumkonzentration führen Berridge und Kollegen auf den Influx von Kalzium entweder aus dem extrazellulärem Raum oder auf die Freisetzung von zellulären Kalziumspeichern zurück (Berridge et al., 2003). Sarco/Endoplasmic-Reticulum Cat ${ }^{2+}$-ATPases1 (SERCA1) scheint hierbei eine zentrale Rolle zu spielen, da es Kalzium auch gegen den Kalziumgradienten in das SR/ER transportiert. Hierbei existieren mindestens zwei Sarco/Endoplasmic-Reticulum $\mathrm{Ca}^{2+}$-ATPase Kanäle (SERCA1 und SERCA2a, (Bhogal und Colyer, 1998)), wovon für die vorliegende Arbeit, aufgrund der Lage des Gens in einer merkmalsassoziierten Region auf SSC3 (Bornemann-Kolatzki, 2004) besonders SERCA1 betrachtet wurde. SERCA1 ist ein Transmembranprotein, bei dem durch alternative splicing entstandene, verschiedene Isoformen bekannt sind (Wuytack et al., 2002). Um ein physiologisches Kalziumniveau in der Zelle aufrecht zu erhalten, wird Kalzium aus dem SR/ER im Wesentlichen durch inositol-1,4,5-posphatreceptor und dem ryanodine-receptor (RyR) freigesetzt (Abb. 4.1; (Barritt, 1999)). Ein verringerter Kalziumgehalt im ER/SR der Zellen kann somit der Grund für eine geringere Sensibilität der Zelle gegenüber apoptoseinduzierender Reize sein (Oakes et al., 2005).

Die in der vorliegenden Arbeit untersuchten Kalziumgehalte beim Schwein lassen sich auf dieser Grundlage durch eine Störung der Kalziumhomöostase oder durch eine gestörte Verteilung des Kalziums erklären. Tanyel legt hierfür beim Menschen eine Störung der Innervation zugrunde (Tanyel et al., 2003). Ein gestörter Kalziumaustausch zwischen SR/ER und den Mitochondrien und eine 
Verringerung der Cytochrom C Sekretion könnte ebenfalls zu einem Ausbleiben der Apoptose führen (Demaurex und Distelhorst, 2003).

Unter Zuhilfenahme des bereits angesprochenen Genomscans, wurden die in der vorliegenden Arbeit untersuchten Kandidatengene aus der Apoptosekaskade bzw. aus den kalziumregulierenden Mechanismen in der Zelle definiert. Bei diesen Genen handelt es sich um CASPASE9, $B A K 1$, programmed cell death 5 (PDCD5), BCl-2-like 11 (BCL2L11), BCl2-like 12 (BCL2L12), caspase recruitment domain family, member 8 (CARD8) und ITPR-3. Dabei konnte in einer älteren Studie eine Beteiligung von SERCA1 an der Vererbung von Hernia i/s als kausales Gen - zumindest in der untersuchten Population - ausgeschlossen werden (Beck, 2008).

\section{Eingliederung der Arbeit in das Forschungsprojekt}

Im Rahmen des Forschungsprojektes „Entwicklung von Techniken und Methoden zur Kartierung von Defektgenen beim Schwein", welches durch den Förderverein Biotechnologieforschung e.V. sowohl finanziell als auch durch die Bereitstellung von Probenmaterial gefördert wird, werden am Tierärztlichen Institut molekulargenetische Untersuchungen zu Hernia i/s durchgeführt.

In der ersten Phase des Projektes wurden mögliche Kandidatengene molekulargenetisch untersucht und mit Hilfe einer Kopplungsanalyse ihr möglicher Bezug zum Defekt Hernia i/s ermittelt. Bornemann-Kolatzki führte ein Genomic mismatch scanning und einen Genomscan mittels polymorpher DNA-Marker durch und konnte dadurch verschiedene Chromosomenregionen, die mit der Vererbung von Hernia $i / s$ in Verbindung stehen, charakterisieren. Nach Abschluss dieses Genomscans lagen Informationen zu den mit dem Defekt Hernia i/s assoziierten Regionen auf den porcinen Chromosomen SSC3, SSC6, SSC7, SSC12 und SSC15 vor (Bornemann-Kolatzki, 2004).

Bei den in Phase 1 ermittelten Kandidatengenen handelt es sich um INSL3 und HOXA10, die als funktionelle Kandidatengene untersucht wurden und deren Beteiligung an der Vererbung von Hernien anhand von Assoziationsstudien an Voll- und Halbgeschwisterpopulationen 
ausgeschlossen werden konnte (Uibeleisen, 2001, Knorr et al., 2004). Weitere untersuchte Kandidatengene sind die Gene der Hyaluronanabbauenden Enzyme (HYAL, HEXB, GUSB) und das CALC/CGRP. Auch diese Gene wurden aufgrund einer fehlenden Merkmalsassoziation und ihrer chromosomalen Lokalisation als funktionelle Kandidatengene zumindest für die untersuchte Population - ausgeschlossen (Mueller et al., 2003, Gatphayak et al., 2004, Beck et al., 2006). GUSB wurde zwar in einer merkmalsassoziierten Region auf SSC 3 kartiert (Beck et al., 2002) konnte jedoch nach SNP-Analysen an Voll- und Halbgeschwisterpopulationen als funktionelles Kandidatengen ausgeschlossen werden (Beck et al., 2006).

Germerodt hat in ihrer Arbeit drei Kandidatengene (PRKAR1A1, ERN1 und SEPT4) aufgrund komparativer Genkarten als funktionelle Kandidatengene auf SSC12 untersucht und konnte eine signifikante Kopplung eines Mikrosatelliten mit dem Defekt Hernia i/s nachweisen (Germerodt, 2009).

In der zweiten Phase des Projektes, in die sich auch die vorliegende Arbeit eingliedert, erfolgt eine weitere Feinkartierung der assoziierten Chromosomenregionen. Hierbei wurden Kandidatengene aus komparativen Genkarten ermittelt, die mit der Vererbung des Defektes in Verbindung zu stehen scheinen und in einer der merkmalsassoziierten Regionen liegen. Zusätzlich wurden physiologische Untersuchungen zum Verschluss des PV aufgrund der unter Punkt 4 beschrieben Zusammenhänge durchgeführt (Beuermann et al., 2009). Hierauf basierend wurden zusätzliche Kandidatengene sowohl aus der apoptotischen Kaskade als auch Kalzium-regulierende Gene in den o. g. Regionen charakterisiert. Eine weitere Untersuchung der Gene wurde über die Mikrosatellitenanalyse (TOMMI) durchgeführt (Germerodt et al., 2008).

\section{Genetik und Physiologie - quo vadis?}

Aufbauend auf einer Kopplungs- und Assoziationsstudie (BornemannKolatzki, 2004, Grindflek et al., 2006) und durch eine vertiefte Kandidatengen-Analyse in den merkmalsassoziierten Genomregionen 
lässt sich zusammenfassend festhalten, dass Gene und DNA-Marker identifiziert wurden, die mit den Defekt Hernia i/s u. U. assoziiert sind. Physiologische Untersuchungen in betroffenen Tieren haben deutliche Unterschiede im Kalziumgehalt der Gewebe des Reproduktionstraktes gezeigt (Beuermann et al., 2009), was wiederum die Auswahl der Kandidatengene beeinflusste und zur Aufklärung der Ursachen für diesen Defekt dienen sollte.

Um dieses komplexe Krankheitsbild, welches von vielen Genen, GenUmwelt-Interaktionen und Gen-Gen-Interaktionen beeinflusst wird, zu klären, wird weit mehr als eine Kandidatengenanalyse nötig sein. Daher sind weitere molekulargenetische und physiologische Untersuchungen zu Defekt Hernia i/s unabdingbar.

Die genaue Untersuchung zur Ätiologie der Hernia i/s stellt die Wissenschaft vor ein großes Problem, da die Prädisposition für diesen Defekt zum Diagnosezeitpunkt abgeschlossen ist. Um jedoch Untersuchungen an den Geweben der betroffenen Tiere durchführen zu können, müssen diese bereits getötet werden, bevor eine eindeutige Diagnose möglich ist. Um diese Diskrepanz zu umgehen, könnte ein Klon aus einem Tier, welches den Defekt Hernia i/s zeigt, als entsprechendes Untersuchungsobjekt dienen. Diese Tiere (Klone) zeigen sicher den defekttypischen Phänotyp, sodass sie für Expressionsuntersuchungen beim DT und Untersuchungen zur Obliteration des PV ein geradezu ideales Tiermodell bilden. Teilweise kontrovers diskutierte Hypothesen zur Ätiologie von Hernia i/s (Tanyel vs Hutson) ließen sich hierdurch u. U. klären.

Eine weitere Möglichkeit der Untersuchung bildet hierbei die Analyse von verschiedenen Zellkulturen, die aus den verschiedenen Geweben um und im Reproduktionstrakt gewonnen werden. Gerade in solchen Zellkulturmodellen lassen sich die Kalziumhomöostase aber auch verschiedenen Apoptosewege genauer untersuchen. Mittels DNAMicroarrays lassen sich verschiedene Genexpressionen in den Zellkulturmodellen untersuchen und durch Protein-Microarrays auch deren Proteinexpression analysieren. Hierbei können auch Interaktionen auf Proteinebene genauer betrachtet werden. Sie ermöglichen darüber 
hinaus auch die Analyse von physiologischen und pathophysiologischen Prozessen molekularer Mechanismen und finden, laut Geldermann bereits Anwendung bei der Krankheitsdiagnostik und der Entwicklung therapeutischer Marker (Geldermann, 2005).

Somit lassen sich Kandidatengene, neben der bereits angesprochenen Kopplungs- und Assoziationsstudie, aus diesen physiologischen und Expressionsanalysen anhand komparativer Genkarten definieren. Die für Ende 2009 geplante Fertigstellung des Sequenzierungsprojektes des Schweinegenoms kann dabei ebenfalls zur Charakterisierung genauer definierter Kandidatengene herangezogen werden.

Beck geht in ihrer Arbeit darauf ein, auch Kandidatengene, die als stark funktionell angesehen werden (z.B. $A R$ und $C G R P$ ), zu untersuchen (Beck, 2008). Diese liegen zwar außerhalb der von Bornemann-Kolatzki definierten Genomregionen (2004), werden hingegen aber von Grindflek und Kollegen (2006) und auch in einer jüngsten Studie von Du (Du et al., 2009) in einer Genomregion lokalisiert, die mit dem Merkmal Hernia i/s assoziiert ist.

Die Entwicklung von Mikrosatelliten (MS) als DNA-Marker zur merkmalsassoziierten Identifikation von Genomregionen ist eine verbreitete Methode und fand über die TOMMI-Methode (Chen et al., 2005) auch in dieser Arbeit ihren Einsatz. Diese MS wurden an betroffenen Halbgeschwisterpopulationen bereits getestet, genauere Analysen zur Kopplung dieser MS mit dem Defekt Hernia i/s stehen hierbei jedoch noch aus. Hierauf aufbauend können Untersuchungen an SNPs in den evtl. assoziierten Genen mittels jüngst vorgestellten Hochdurchsatz-Sequenzierungsmethoden vorgenommen werden. Diese einfachen und oftmals kostengünstigen Methoden erlauben eine hochparallele Detektion von Polymorphismen in den Kandidatengenen und sind somit für die Genomanalyse und bei der Aufklärung von komplex-vererbten Krankheiten von hohem Nutzen (Schuetz, 2009). 


\section{Literaturverzeichnis}

ADHAM, I. M., EMMEN, J. M. \& ENGEL, W. (2000): The role of the testicular factor INSL3 in establishing the gonadal position. Mol Cell Endocrinol, 160, 11-6.

ALTHOFF, W. (1985): Zur erblichen Abhängigkeit verschiedener Geburtsfehler beim Schwein. Dissertation, Universität Bomm.

ALTHOFF, W., MAYER, M., RICHTER, L. \& SIMON, D. (1988): Zur erblichen Abhängigkeit der Geburtsfehler Brüche und Binnenhodigkeit beim Schwein. Züchtungskunde, 319-329.

AMANN, R. P. \& VEERAMACHANENI, D. N. (2007): Cryptorchidism in common eutherian mammals. Reproduction, 133, 541-61.

BARRITT, G. J. (1999): Receptor-activated Ca2+ inflow in animal cells: a variety of pathways tailored to meet different intracellular $\mathrm{Ca} 2+$ signalling requirements. Biochem J, 337 (Pt 2), 153-69.

BARTHOLD, J. S. \& REDMAN, J. F. (1996): Association of epididymal anomalies with patent processus vaginalis in hernia, hydrocele and cryptorchidism. J Urol, 156, 2054-6.

BECK, J. (2008): Genomanalyse beim landwirtschaftlichen Nutztier. Dissertation, GeorgAugust-Universität Göttingen.

BECK, J., BORNEMANN-KOLATZKI, K., KNORR, C., TAEUBERT, H. \& BRENIG, B. (2006): Molecular characterization and exclusion of porcine GUSB as a candidate gene for congenital hernia inguinalis/scrotalis. BMC Vet Res, 2, 14.

BECK, J., KNORR, C., HABERMANN, F., FRIES, R. \& BRENIG, B. (2002): Assignment of the beta-glucuronidase (GUSB) gene to porcine chromosome SSC3p16-->p14 by FISH and confirmation by hybrid panel analyses. Cytogenet Genome Res, 97, $277 \mathrm{G}$.

BEISSNE, B. (2003): Genetische Analyse von angeborenen Anomalien bei den Nachkommen von Besamungsebern. Dissertation, Tierärztliche Hochschule Hannover.

BERGE, S. (1941): The inheritance of paralysed hind legs, scrotal hernia and atresia ani in pigs. $J$ Hered, 271-274.

BERGNER, A. \& HUBER, R. M. (2008): Regulation of the endoplasmic reticulum $\mathrm{Ca}(2+)-$ store in cancer. Anticancer Agents Med Chem, 8, 705-9.

BERRIDGE, M. J., BOOTMAN, M. D. \& RODERICK, H. L. (2003): Calcium signalling: dynamics, homeostasis and remodelling. Nat Rev Mol Cell Biol, 4, 517-29.

BEUERMANN, C., BECK, J., SCHMELZ, U., DUNKELBERG, H., SCHUTZ, E., BRENIG, B. \& KNORR, C. (2009): Tissue calcium content in piglets with inguinal or scrotal hernias or cryptorchidism. J Comp Pathol, 140, 182-6.

BHOGAL, M. S. \& COLYER, J. (1998): Depletion of sarcoplasmic reticulum calcium prompts phosphorylation of phospholamban to stimulate store refilling. Ann $N Y$ Acad Sci, 853, 260-3.

BICKEBÖLLER, H. \& FISCHER, C. (2007): Einführung in die genetische Epidimiologie, Springer Verlag.

BORNEMANN-KOLATZKI, K. (2004): Durchführung eines Genomscans mit polymorphen DNA-Markern und Genomic Mismatch Scanning (GMS) bei Sus scrofa zur Detektion Hernia inguinalis/scrotalis assoziierter Genomregionen. Dissertation, Tierärztliche Hochschule Hannover \& Georg-August-Universität Göttingen.

BUSCHBELL, H., THOLEN, E. \& WIESE, M. (2007): Neue Zuchtwertschätzung für Erbfehler. TopGenetik, GFS. 
CAIN, M. P., KRAMER, S. A., TINDALL, D. J. \& HUSMANN, D. A. (1994): Alterations in maternal epidermal growth factor (EGF) effect testicular descent and epididymal development. Urology, 43, 375-8.

CHEN, K., KNORR, C., BORNEMANN-KOLATZKI, K., REN, J., HUANG, L., ROHRER, G. A. \& BRENIG, B. (2005): Targeted oligonucleotide-mediated microsatellite identification (TOMMI) from large-insert library clones. BMC Genet, 6, 54.

CLARNETTE, T. D. \& HUTSON, J. M. (1999): Exogenous calcitonin gene-related peptide can change the direction of gubernacular migration in the mutant trans-scrotal rat. $J$ Pediatr Surg, 34, 1208-12.

CLARNETTE, T. D., LAM, S. K. \& HUTSON, J. M. (1998): Ventriculo-peritoneal shunts in children reveal the natural history of closure of the processus vaginalis. $J$ Pediatr Surg, 33, 413-6.

DEMAUREX, N. \& DISTELHORST, C. (2003): Cell biology. Apoptosis--the calcium connection. Science, 300, 65-7.

DENIAUD, A., SHARAF EL DEIN, O., MAILLIER, E., PONCET, D., KROEMER, G., LEMAIRE, C. \& BRENNER, C. (2008): Endoplasmic reticulum stress induces calciumdependent permeability transition, mitochondrial outer membrane permeabilization and apoptosis. Oncogene, 27, 285-99.

DU, Z. Q., ZHAO, X., VUKASINOVIC, N., RODRIGUEZ, F., CLUTTER, A. C. \& ROTHSCHILD, M. F. (2009): Association and haplotype analyses of positional candidate genes in five genomic regions linked to scrotal hernia in commercial pig lines. PLOS ONE, 4, e4837.

DÖPFER, R. (2009) Persönliche Gespräche.

GATPHAYAK, K., KNORR, C., BECK, J. \& BRENIG, B. (2004): Molecular characterization of porcine hyaluronidase genes 1,2 , and 3 clustered on SSC13q21. Cytogenet Genome Res, 106, 98-106.

GELDERMANN, H. (2005): Tier-Biotechnologie, Stuttgard, 2005, Eugen Ulmer Verlag.

GERMERODT, M. (2009): Molekulargenetische Untersuchungen zur Verbesserung der männlichen Fruchtbarkeit und Bekämpfung des erbdefektes Hernia inguinalis/scrotalis in der Schweinezucht. Georg-August-Universität Göttingen.

GERMERODT, M., BEUERMANN, C., ROHRER, G. A., SNELLING, W. M., BRENIG, B. \& KNORR, C. (2008): Characterization and linkage mapping of 15 porcine STS markers to fine-map chromosomal regions associated with hernia inguinalis/scrotalis. Anim Genet, 39, 671-2.

GRINDFLEK, E., MOE, M., TAUBERT, H., SIMIANER, H., LIEN, S. \& MOEN, T. (2006): Genome-wide linkage analysis of inguinal hernia in pigs using affected sib pairs. BMC Genet, 7, 25.

HERES, S. (1976) Research results in congenital hernias of pigs. Proceeding of the Fourth International Pig Veterinary Society Congress. Iower State University, Ames.

HEYNS, C. F., HUMAN, H. J., WERELY, C. J. \& DE KLERK, D. P. (1990): The glycosaminoglycans of the gubernaculum during testicular descent in the fetus. $J$ Urol, 143, 612-7.

HUTSON, J. M. (1985): A biphasic model for the hormonal control of testicular descent. Lancet, 2, 419-21.

HUTSON, J. M., ALBANO, F. R., PAXTON, G., SUGITA, Y., CONNOR, R., CLARNETTE, T. D., GRAY, A. Z., WATTS, L. M., FARMER, P. J. \& HASTHORPE, S. (2000): In vitro fusion of human inguinal hernia with associated epithelial transformation. Cells Tissues Organs, 166, 249-58.

HUTSON, J. M. \& HASTHORPE, S. (2005): Testicular descent and cryptorchidism: the state of the art in 2004. J Pediatr Surg, 40, 297-302. 
HUTSON, J. M., HASTHORPE, S. \& HEYNS, C. F. (1997): Anatomical and functional aspects of testicular descent and cryptorchidism. Endocr Rev, 18, 259-80.

KNORR, C. (2005): Genome, Gene und molekulare Marker in der modernen Tierzucht. Habilitation, Georg-August-Universität Göttingen.

KNORR, C., TAUBERT, H., PETERS, U. \& BRENIG, B. (2004): Characterization of two SNPs (single nucleotide polymorphisms) in the porcine INSL3 gene and their exclusion as a common genetic basis of hernia inguinalis in pigs. Biochem Genet, 42, 11-9.

LEVY, J. B. \& HUSMANN, D. A. (1995): The hormonal control of testicular descent. J Androl, 16, 459-63.

LIPSKAIA, L., HULOT, J. S. \& LOMPRE, A. M. (2009): Role of sarco/endoplasmic reticulum calcium content and calcium ATPase activity in the control of cell growth and proliferation. Pflugers Arch, 457, 673-85.

MATTSON, M. P. \& CHAN, S. L. (2003): Calcium orchestrates apoptosis. Nat Cell Biol, 5, 1041-3.

MCMAHON, D. R., KRAMER, S. A. \& HUSMANN, D. A. (1995): Antiandrogen induced cryptorchidism in the pig is associated with failed gubernacular regression and epididymal malformations. $J$ Urol, 154, 553-7.

MIKAMI, H. \& FREDEEN, H. T. (1979): A genetic study of cryptorchidism and scrotal hernia in pigs. Can J Genet Cytol, 21, 9-19.

MUELLER, A., KNORR, C., HABERMANN, F., SLANCHEV, K., ZWILLING, D., FRIES, R. \& BRENIG, B. (2003): Assignment of the beta-N-acetylhexosaminidase gene (HEXB) to porcine chromosome SSC2q21-->q22 by fluorescence in situ hybridization and by analysis of somatic cell and radiation hybrid panels. Cytogenet Genome Res, $101,178$.

NEF, S. \& PARADA, L. F. (1999): Cryptorchidism in mice mutant for Insl3. Nat Genet, 22, 295-9.

OAKES, S. A., SCORRANO, L., OPFERMAN, J. T., BASSIK, M. C., NISHINO, M., POZZAN, T. \& KORSMEYER, S. J. (2005): Proapoptotic BAX and BAK regulate the type 1 inositol trisphosphate receptor and calcium leak from the endoplasmic reticulum. Proc Natl Acad Sci U S A, 102, 105-10.

ORRENIUS, S., ZHIVOTOVSKY, B. \& NICOTERA, P. (2003): Regulation of cell death: the calcium-apoptosis link. Nat Rev Mol Cell Biol, 4, 552-65.

RIZZUTO, R., PINTON, P., FERRARI, D., CHAMI, M., SZABADKAI, G., MAGALHAES, P. J., DI VIRGILIO, F. \& POZZAN, T. (2003): Calcium and apoptosis: facts and hypotheses. Oncogene, 22, 8619-27.

ROTHSCHILD, M. F., HU, Z. L. \& JIANG, Z. (2007): Advances in QTL mapping in pigs. Int J Biol SCi, 3, 192-7.

SATOKATA, I., BENSON, G. \& MAAS, R. (1995): Sexually dimorphic sterility phenotypes in Hoxa10-deficient mice. Nature, 374, 460-3.

SCHUETZ, E. (2009): Molecular Diagnostics based on Melting Transition Curves. Habilitation, Georg-August-Universität Göttingen.

SILVA-RAMOS, M., OLIVEIRA, J. M., CABEDA, J. M., REIS, A., SOARES, J. \& PIMENTA, A. (2006): The CAG repeat within the androgen receptor gene and its relationship to cryptorchidism. Int Braz J Urol, 32, 330-4; discussion 335.

STIGLER, J. (1990): Analyse von Erbfehlern beim Schwein. Dissertation, LudwigMaximilian-Universität München.

TANYEL, F. C., ERDEM, S., BUYUKPAMUKCU, N. \& TAN, E. (2002): Smooth muscle within incomplete obliterations of processus vaginalis lacks apoptotic nuclei. Urol Int, 69, 42-5.

TANYEL, F. C. \& OKUR, H. D. (2004): Autonomic nervous system appears to play a role in obliteration of processus vaginalis. Hernia, 8, 149-54. 
TANYEL, F. C., ULUSU, N. N., TEZCAN, E. F. \& BUYUKPAMUKCU, N. (2003): Total calcium content of sacs associated with inguinal hernia, hydrocele or undescended testis reflects differences dictated by programmed cell death. Urol Int, 70, 211-5.

TEZ, M. \& KILIC, Y. A. (2006): Stem cell origin theory of the inguinal hernia. Med Hypotheses, 66, 1042-3.

UIBELEISEN, A.-C. (2001): Isoloierung, Charackterisierung und molekulargenetische Analysen des porcinen HOXA10 Gens. Masterarbeit, Georg-August-Universität Göttingen.

ULUSU, N. N., TANDOGAN, B. \& TANYEL, F. C. (2007): Sarco(endo)plasmic reticulum and plasmalemmal $\mathrm{Ca}(2+)$-ATPase activities in cremaster muscles and sacs differ according to the associated inguinal pathology. Cell Biochem Funct, 25, 515-9.

WALDMANN, K.-H. \& WENDT, M. (2001): Lehrbuch der Schweinekrankheiten, Paul Parey Verlag.

WENSING, C. J. (1986): Testicular descent in the rat and a comparison of this process in the rat with that in the pig. Anat Rec, 214, 154-60.

WENSING, C. J. (1988): The embryology of testicular descent. Horm Res, 30, 144-52.

WILLIAMS, M. P. L. \& HUTSON, J. M. (1991): The phylogeny of testicular descent. Pediatris Surgery International, 6, 162-166.

WUYTACK, F., RAEYMAEKERS, L. \& MISSIAEN, L. (2002): Molecular physiology of the SERCA and SPCA pumps. Cell Calcium, 32, 279-305.

ZDS, J. (2007): Schweineproduktion 2007 in Deutschland, Ausgabe 2008.

ZHONG, F., DAVIS, M. C., MCCOLL, K. S. \& DISTELHORST, C. W. (2006): Bcl-2 differentially regulates $\mathrm{Ca} 2+$ signals according to the strength of $\mathrm{T}$ cell receptor activation. J Cell Biol, 172, 127-37.

ZIMMERMANN, S., STEDING, G., EMMEN, J. M., BRINKMANN, A. O., NAYERNIA, K., HOLSTEIN, A. F., ENGEL, W. \& ADHAM, I. M. (1999): Targeted disruption of the Insl3 gene causes bilateral cryptorchidism. Mol Endocrinol, 13, 681-91. 


\section{Danksagung}

Ich bedanke mich besonders bei Herrn Prof. Dr. Dr. Bertram Brenig für die Überlassung des Themas und für die Betreuung meiner Dissertation. Weiterhin gilt mein besonderer Dank Herrn Prof. Dr. Wolfgang Holtz für die Übernahme des Koreferrats und Herrn Prof. Dr. Christoph Knorr für die Betreuung meiner Arbeit. Herrn PhD Dr. Ekkehard Schütz danke ich für die geduldige Unterstützung.

Meinen Kollegen und Koautoren danke ich für die gute Zusammenarbeit. Besonders erwähnen möchte ich dabei Frau Dr. Julia Beck. Ohne Jule wäre dies alles nicht möglich gewesen - DANKE!

Außerdem danke ich der HUMANCHEMIE GmbH für die Möglichkeit, meine Dissertation neben der Tätigkeit bei den „Humanchemikern" zu beenden.

Zuletzt möchte ich mich bei meiner Familie bedanken, meinen Eltern Erich und Sabine Beuermann, die mich immer unterstützt haben. Und danke auch Euch beiden, Viola und Emma, ...für alles. 


\title{
8 Lebenslauf
}

\section{Zu meiner Person}

\author{
geboren am 22. März 1978 in Göttingen \\ verheiratet, eine Tochter. \\ Ausbildung \\ 1998-2005 Agrarwissenschaftsstudium an der Georg-August- \\ Universität Göttingen Schwerpunkt: Tierproduktion. \\ Masterarbeit im Tierärztlichen Institut mit dem Thema: \\ „Molekulare Charakterisierung der porcinen 37 kDa \\ Laminin-Rezeptor-Genfamilie". \\ Abschluss: Master of Science in Agriculture \\ (M.Sc.agr.). \\ 1997-1998 Grundwehrdienst in Rheine. \\ 1997 Grotefendt-Gymnasium Hann. Münden: Abitur.
}

\section{Beruflicher Werdegang}

ab 02.2007 Verkaufsleiter und Assistent der Geschäftsleitung der HUMANCHEMIE GmbH Alfeld.

seit 2006 Angestellt im Tierärztlichen Institut Göttingen im Rahmen der Promotion zum Thema: „Molekulargenetische und physiologische Untersuchungen zur Vererbung des Erbdefektes Hernia inguinalis/scrotalis beim Schwein".

\section{Kenntnisse \& Fähigkeiten}

EDV: Mac OS X und Windows, fundierte Kenntnisse in Word, Excel, PowerPoint, Adobe Photoshop und Access.

Fremdsprachen: Englisch in Wort und Schrift, Französisch, Spanisch (Grundkenntnisse).

Führerschein: B-C-E. 